\title{
Macrophage cell lines and murine infection by Salmonella Typhi
}

\section{L-form bacteria}

\author{
*Debayan Ganguli, *Swarnali Chakraborty, Suparna Chakraborty, Ananda Pal, Animesh Gope \\ \#Santasabuj Das
}

ICMR- National Institute of Cholera and Enteric diseases, P-33, CIT Rd, Subhas Sarobar Park, Phool Bagan, Beleghata, Kolkata, West Bengal 700010

* Contributed equally

\# Corresponding author Email: santasabujdas@yahoo.com

Keywords: Salmonella, L- form, Ampicillin, Macrophage

\begin{abstract}
Antibiotic resistance of pathogenic bacteria has emerged as a major threat to public health worldwide. While stable resistance due to the acquisition of genomic mutations or plasmids carrying antibiotic-resistance genes is well-established, much less is known about the temporary and reversible resistance induced by antibiotic treatment, such as the one due to treatment with bacterial cell-wall inhibiting antibiotics like ampicillin. Typically, ampicillin concentration in the blood and other tissues gradually increases over time after initiation of thetreatment. As a result, the bacterial population is exposed to a concentration gradient of ampicillin. This is different from in vitro drug testing where the organism is exposed to fixed drug concentrations from the beginning till the end. To mimic the mode of antibiotic exposure of microorganisms in the tissues, we cultured the wild type, ampicillin-sensitive Salmonella Typhi Ty2 strain (S. Typhi Ty2) in the presence of increasing concentrations of ampicillin over a period of 14 days. This resulted in the development of a strain that exhibited several features of the so-called L- form of bacteria, such
\end{abstract}


as the absence of cell wall, altered shape and slower growth rate compared with the parental strain. Studies on the pathogenesis of $S$. Typhi L-form showed efficient infection of the murine and human macrophage cell lines. More importantly, $S$. Typhi L-form was also able to establish infection in a mouse model to the extent comparable to its parental strain. These results suggested that L-form generation following initiation of antibiotic treatment could lead to drug escape of $S$. Typhi and direct spread to new cells (macrophages), which sustain the infection. Oral infection by the L-form bacteria underscores the potential of rapid disease transmission through faeco-oral route, highlighting the need for new approaches to decrease the reservoir of infection.

\section{Introduction}

Around 26.9 million cases of typhoid fever are reported annually worldwide [1]. Multi drug resistant strains of Salmonella Typhi are known for several decades and were isolated at different parts of the globe. $S$. Typhi was earlier treated with Ampicillin, chloramphenicol and trimethoprim-sulfamethoxazole [2]. Appearance of multidrug resistance to the first line antibiotics during late 1980s led fluoroquinolones, especially ciprofloxacin to become the drug of choice for typhoid fever [3]. In 1991, S. Typhi strain resistant to ciprofloxacin emerged, which lead to an outbreak in 1997 [4]. Subsequently, macrolides, $3^{\text {rd }}$ generation cephalosporins and carbapenems were widely used to treat $S$. Typhi infection. In 2018 and 2019, extreme drug resistant (XDR) $S$. Typhi strains, unresponsive to macrolides, fluoroquinolones and $3^{\text {rd }}$ generation of cephalosporins were reported from Hyderabad [5, 6], India and Pakistan. WHO had recently announced 10,365 infections with XDR strains in Pakistan and travel-associated infections in China, USA, Australia, Denmark and Canada, thus making $S$. Typhi drug resistance a global threat [7]. Interestingly, reversal to ampicillin sensitivity of $S$. Typhi was reported by studies carried out at different parts of India [8]. Harish et al, 2011 had reported that ampicillin-resistant strains in Chennai fell from $52 \%$ to $23 \%$ during the period 2002 to 2009 [9]. In another study, around $94.5 \%$ of S. Typhi clinical isolates collected from India were found to be ampicillin sensitive [10]. 
Antibiotic resistance of $S$. Typhi was believed to be plasmid borne. Genome sequencing of CT18 and several other Ampicillin resistant $S$. Typhi strains revealed the presence of Salmonella- associated transferable R plasmid, called pHCM1 [11]. This plasmid was responsible for the resistance to all three first-line antibiotics. However, temporary and reversible ampicillin resistance may also be conferred by the formation of L-form bacteria. Ampicillin targets the bacterial cell wall machinery and bacteria can overcome the lethal effects of the drug by shutting down the cell wall production temporarily. Bacterial L-form was first discovered in 1935 [12] and subsequent studies characterized this form. However, most studies were carried out in the pre-molecular biology era and thorough knowledge about the formation of the L form is lacking. Recently, Errington and others explored the mechanism underlying Lform transition of $B$. subtilis $[13,14,15]$. They discovered that deletion of the cell wall machinery genes (MurE operon) was not sufficient to induce stable L-form growth. Inactivating mutation of ispA or a glycolytic or respiratory chain enzyme in addition to MurE knock out was necessary for the L-form to survive [15]. However, the transient and reversible nature of antibiotic resistance associated with the L-form failed to attract much global attention, leading to poor understanding about its significance in pathogenesis and contribution to treatment failures associated with antimicrobial resistance. Notwithstanding, bacterial L-forms were shown to be responsible for recurrent and chronic infections, although their diagnosis as etiologic agents is frequently missed due to slow growth and the requirement for specialized culture medium. Back in 1965, L-form was suggested to cause chronic Staphylococcal infection [16]. Gutman et al. isolated L-forms of Escherichia coli, Klebsiella spp. and Enterococcus faecalis from patients suffering from chronic bacteriuria or pyelonephritis [17]. E. facealis L-forms persisted in rats infected with walled bacteria, followed by treatment with penicillin [18]. Cell wall deficient L-forms of Corynaebacterium and Mycobacterium tuberculosis were isolated from blood samples of patients suffering from subacute bacterial endocarditis and sarcoidosis, respectively who received antibiotic treatment $[19,20]$. Similar 
observations were made for patients with chronic brucellosis [21] and rheumatic fever caused by group A streptococci [22].

Salmonella was also reported to undergo L-form transition in the presence of cell wall-targeting drugs. Recent studies with $S$. Typhimurium showed that L-form can be readily induced by cefazolin [23]. These L form bacteria were also resistant to $3^{\text {rd }}$ generation cephalosporin [23]. Others envisaged L-form Salmonella to be responsible for the long term effects of attenuated bacterial vaccines, indicating their non-pathogenic nature [24]. On the contrary, several studies from 1970s suggested that L-form Salmonella was capable to cause disease. However, most of these publications were either not in English or unavailable in the databases.Development of Lform Salmonella in vivo and its role in pathogenesis require further elucidation

In this study, we induced transition to $S$. Typhi L-form in vitro by treating an ampicillinsensitive strain (Ty2) with increasing concentrations of ampicillin over a period of 14 days. This exposure of a drug-sensitive $S$. Typhi strain to a gradient of ampicillin would mimic the in vivo condition during treatment of typhoid patients with antibiotics. We characterized the $S$. Typhi L-form and demonstrated its abilities to infect murine and human macrophage cell lines. Finally, we showed that L-form bacteria was capable to establish productive infection in a mouse model. This study, for the first time, explored in detail the potential pathogenic role of ampicillin-induced L-form of $S$. Typhi.

\section{MATERIALS AND METHODS}

\section{Cells and reagents.}

Cell lines and cell culture reagents were procured from the American Type Culture Collection (ATCC) and Invitrogen, respectively. Bacterial culture media were purchased from BD Difco.

\section{Generation of $S$. Typhi L-form}

Ampicillin sensitive $S$. Typhi Ty2 strain was sequentially exposed to increasing concentrations 
of ampicillin $(0.05 \mathrm{ug} / \mathrm{ml}$ to $200 \mathrm{ug} / \mathrm{ml})$ over a period of 14 days. Bacteria were allowed to grow for two days at each concentration of ampicillin before being transferred to media containing the next higher concentration of ampicillin.

\section{In vitro growth measurement}

Growth curves of the parental and $\mathrm{A}_{200}$ strains were obtained by plotting optical densities of the bacterial culture media taken at $600 \mathrm{~nm}$ wavelength $\left(\mathrm{OD}_{600}\right)$ after different time intervals from the beginning of the culture.

\section{LPS extraction}

LPS was purified by hot phenol method and was quantified by phenol-sulphuric acid method as described previously [25]. Briefly, bacteria were allowed to grow for $18 \mathrm{hrs}$ in LB medium. The harvested cells were re-suspended in distilled water and lysed by sonication ( 7 Watt, 10 seconds pulse and 10 seconds interval for 20 mins). The cell lysates produced were treated with DNaseI $(10 \mathrm{mg} / \mathrm{ml})$ and RNase $(5 \mathrm{mg} / \mathrm{ml})$, followed by proteinase $\mathrm{K}(20 \mathrm{mg} / \mathrm{ml})$ at $60^{\circ} \mathrm{C}$ for 1 hr. The lysate was then incubated with phenol at $65^{\circ} \mathrm{C}-70^{\circ} \mathrm{C}$ with vigorous shaking for $1 \mathrm{hr}$ and the solution was allowed to cool down at $10^{\circ} \mathrm{C}$ before being centrifuged at $10000 \mathrm{rpm}$ for10 min to separate the organic and aqueous phases. After that, double volume of ethanol was added to the aqueous phase and the solution was incubated overnight at $-20^{\circ} \mathrm{C}$ for complete precipitation of the LPS.

\section{Peptidoglycan estimation}

Peptidoglycan was measured according to the standard protocol [26]. One hundred microliter of bacterial culture was incubated with $50 \mu \mathrm{L}$ of $1 \mathrm{M} \mathrm{NaOH}$ at $37{ }^{\circ} \mathrm{C}$ for $30 \mathrm{~min}$, followed by the addition of $1 \mathrm{~mL}$ of concentrated sulphuric acid and incubation in a boiling water bath for $3 \mathrm{~min}$. The mixture was cooled down rapidly on ice for $1 \mathrm{~min}$ and treated with $10 \mu \mathrm{L}$ of 0.16 M Copper sulphate and $20 \mu \mathrm{L}$ of $0.09 \mathrm{M}$ p-hydroxy biphenyl at $30^{\circ} \mathrm{C}$ for $30 \mathrm{~min}$. Peptidoglycan was estimated by taking absorbance at $570 \mathrm{~nm}$ using a spectrophotometer. 


\section{Cell culture}

The human acute monocytic leukemia cell line, THP-1 was maintained in RPMI 1640 medium supplemented with $10 \%$ (vol/vol) heat-inactivated fetal bovine serum (FBS) and penicillinstreptomycin $(50 \mu \mathrm{g} / \mathrm{ml}$ penicillin and $50 \mu \mathrm{g} / \mathrm{ml}$ streptomycin). Cells were differentiated for 24 $\mathrm{hr}$ in the above culture medium, containing $100 \mathrm{nM}$ phorbol 12-myristate 13-acetate (PMA). Experimentation with the differentiated THP-1 cells was done after an additional $24 \mathrm{hr}$ culture in absence of PMA. Mouse macrophage cell line RAW264.7 was cultured in Dulbecco's modified Eagle medium (DMEM) supplemented with 10\% FBS and penicillin-streptomycin.

\section{Gentamicin protection assay:}

This assay was done following the standard procedure [27]. Briefly, THP-1 cells were differentiated in 24-well culture plates $(5 \times 105$ cells/well). RAW264.7 cells were similarly cultured in identical plates. Cells were infected at a multiplicity of infection (MOI) of 50 with $S$. Typhi, opsonized with the respective cell culture medium without antibiotics and were synchronized with the bacteria by centrifugation at $400 \times \mathrm{g}$ for $5 \mathrm{~min}$. Infection was continued for $30 \mathrm{~min}$ at $37^{\circ} \mathrm{C}$ in the presence of $5 \% \mathrm{CO} 2$. Extracellular bacteria were removed by repeated washing with $1 \times$ phosphate-buffered saline (PBS), followed by culturing the cells in media containing $100 \mu \mathrm{g} / \mathrm{ml}$ of gentamicin for $1 \mathrm{~h}$ and then, $15 \mu \mathrm{g} / \mathrm{ml}$ of gentamicin until the time of experiment. Cells were lysed with $1 \times$ PBS containing $0.25 \%$ Triton X-100, and intracellular CFU counts were carried out by overnight growth of the bacteria recovered from the cells on a Luria Agar, plate containing suitable antibiotics.

\section{Confocal microscopy}

THP1 and RAW264.7 cells seeded on collagen-coated coverslips placed in 24 well plates (5x $10^{5}$ cells per well) were infected with $S$. Typhi. One day before the infection, the culture media were replaced by antibiotic-free media, containing dextran-conjugated Alexa fluor 594 $(5 \mathrm{mg} / \mathrm{ml})$ (Thermo Fisher). After three washes with $1 \times$ PBS, coverslips were mounted on clean 
glass slides with fluorophore mounting media (Sigma ). Dextran taken up by the cells emitted red fluorescence from the acidic vacuoles. Stained cells were viewed under a Zeiss LSM 710 confocal microscope, and bacteria were counted manually.

\section{In vivo experiments}

All animal experiments were carried out following the protocols approved by the Institutional Animal Ethics Committee of ICMR-National Institute of Cholera and Enteric Diseases (NICED), Kolkata, India (Project license \# PRO/103/May 2014-September 2017, Date 11.03.1999). ICMR-NICED adheres to the animal handling protocols, issued by the Committee for the Purpose of Control and Supervision of Experiments on Animals (CPCSEA), Ministry of Environment, Forest and Climate Change, Government of India.

An iron-overload mouse model was used for oral $S$. Typhi infection. Briefly, 6- to 8-weeks-old female $\mathrm{BALB} / \mathrm{c}$ mice were injected intraperitoneally with desferrioxamine (Novartis, Switzerland; $0.025 \mathrm{mg} / \mathrm{gm}$ of body weight $)$ and $\mathrm{Fe}^{3+}(0.32 \mathrm{mg} / \mathrm{gm}$ of body weight $) 4 \mathrm{hr}$ prior to the infection. Gastric acid was neutralized with $5 \%$ sodium bicarbonate 20 min before the oral infection with bacterial cultures, which were grown till $\mathrm{OD}_{600}$ reached around 1.0. Mice were infected orally with a sub lethal dose $\left(5 \times 10^{5} \mathrm{CFU}\right)$ of $S$. Typhi and systemic infection was analyzed by the recovery of live bacteria from the liver, spleen, gall bladder, MLN and Peyer's Patches on 2, 4 and 8 post-infection days. To this end, single-cell suspensions were prepared after the organs were lysed with $0.25 \%$ Triton X-100 (Sigma-Aldrich, USA) and plated on LA, containing suitable antibiotics.

For intra-peritonial infection, a sub lethal dose of $S$. Typhi $\left(5 \times 10^{4} \mathrm{CFU}\right)$ was injected into the peritoneum of 6- to 8- weeks old BALB/c mice. Visceral organs (liver, spleen, and gall bladder) were collected at the same time points as above and CFU counts were done as stated before. 


\section{Results}

\section{S. Typhi L-form induction and characterization}

Parental S. Typhi Ty2 strain was subjected to increasing concentrations of ampicillin over a period of 14 days. The bacterial strain obtained was called $A_{200}$, since the dose escalation and subsequent culture was done in the presence of $200 \mathrm{ug} / \mathrm{ml}$ of Ampicillin. Visualization of the $\mathrm{A}_{200}$ strain under scanning electron microscope revealed gross morphological changes in the form of an elongated shape with higher cytoplasmic to nuclear volume (Fig. 1A and 1B). Each bacterial cell of the $\mathrm{A}_{200}$ strain contained more than one nucleoid region as compared to a single nucleoid in the parental strain (compare Fig. S1A and S1B), indicating compromised cell division in the former strain. Furthermore, comparison of the growth curves of the $A_{200}$ and parental Ty2 strain showed significantly reduced growth for $\mathrm{A}_{200}$ strain (Fig. 1C). In contrast, naturally ampicillin-resistant $S$. Typhi strain, CT18 displayed a growth pattern similar to the parental Ty2 strain. However, when $\mathrm{A}_{200}$ was allowed to grow in the absence of ampicillin, it resumed the growth rate of the parental bacteria (Fig. S1C). In addition, most cells rapidly reverted back to the original morphology upon removal of ampicillin (compare Fig. $1 \mathrm{D}$ and 1 E) Time kinetic studies revealed that majority of the $A_{200}$ bacteria assumed the parental morphology with concomitant resumption of the growth rate to that of parental strain within a period of $4 \mathrm{hrs}$ of culture in the absence of ampicillin (Fig. S1D). Together the above observations strongly suggested that $\mathrm{A}_{200}$ strain displayed the characteristics of the L-form bacteria. In agreement with the previous studies [28], L-form $S$. Typhi produced significantly reduced amounts of peptidoglycan (Fig. 1F) and LPS (Fig. S2A) than the parental strain. Similar to other L-form bacteria, $A_{200}$ strain was more resistant to several antibiotics (Fig. S2B), further suggesting that it was indeed the L-form of Salmonella Typhi.

\section{S. Typhi L-form was capable to infect macrophage cells}

To compare infectivity of the parental Ty2 and the $\mathrm{A}_{200}$ strain, mouse macrophage cell line, 
RAW264.7 and human THP-1 cell-derived macrophages were infected with a multiplicity of infection (MOI) of 50, followed by gentamycin protection assay. At the $0 \mathrm{hr}$ time point, higher number of intracellular $\mathrm{A}_{200}$ strain was recovered, indicating greater infectivity (Fig. S3 A and B). Twenty-four hours later, intracellular bacterial number increased for both the strains with $\mathrm{A}_{200}$ remaining more numerous, underscoring that the latter survived and multiplied within the mouse and human macrophage cell lines with equal efficiency as the parental Ty2 strain (Fig S3A and S3B). To gain further insights into the infectivity of $A_{200}$ strain, confocal microscopy was performed. At both $0 \mathrm{hr}$ and $24 \mathrm{hr}$ time points, intracellular parental and $\mathrm{A}_{200}$ strains were co-localized with the acidic vacuoles (stained with Dextran-Alexa fluor 594) (Fig. 2, 3, 4, 5). Together the above results indicated that $\mathrm{A}_{200}$ strain efficiently infected macrophage cells and replicated within acidic vacuoles despite its altered morphology and slower growth in vitro. Given the higher infectivity of $\mathrm{A}_{200}$ strain, we investigated if it resulted in greater cell death. To this end, infected cells were stained with Annexin-V and propidium iodide (PI) and apoptosis was studied by flow cytometry. We observed that $65 \%$ of the Ty2-infected cells were apoptotic at $24 \mathrm{hrs}$ post-infection, as compared with only $11 \%$ apoptosis for $\mathrm{A}_{200}$ infection. At $48 \mathrm{hrs}$ post-infection, apoptosis further increased for Ty2- $(\sim 70 \%)$, but not for $\mathrm{A}_{200}$-infected cells (Fig. 6 A - J). The results indicated that $A_{200}$ strain induced minimal cell death despite more efficient infection in vitro.

\section{$S$. Typhi L-form established infection in mouse model}

The ability of $\mathrm{A}_{200}$ strain to infect macrophage cell lines prompted us to investigate if the strain was also capable to establish infection in vivo. Mice were infected orally as well as through the intraperitoneal (IP) route with a sub-lethal dose of the parental Ty2 or $\mathrm{A}_{200}$ strain. Bacterial load was measured at 2, 4 and 8 days post-infection (dpi) in the visceral organs. Equal and high number of both the bacterial strains were recovered from the liver, spleen and gall bladder after 2 days of intraperitoneal infection, indicating that $\mathrm{A}_{200}$ was equally competent as the parental 
strain to infect live animals (Fig. 7). Bacterial load further increased for both strains at 4 dpi, underscoring similar efficiency of survival and replication in vivo. However, there was a drastic reduction of load in the visceral organs at $8 \mathrm{dpi}$ that suggested pathogen clearance by the host immune system. Interestingly, the visceral load for A200 was significantly higher compared with the parental strain at the later time point. This observation indicated that $\mathrm{A}_{200}$ strain was perhaps more competent in evading the host immune system. As opposed to intraperitoneal infection, substantial bacterial recovery after oral administration was only observed at 4 dpi, suggesting delay in systemic invasion following intestinal colonization (Fig 8). Moreover, significantly fewer $\mathrm{A}_{200}$ bacteria than the parental strain were recovered from the internal organs at this time point. Given that both the strains survived and replicated in the visceral organs with equal competence and $\mathrm{A}_{200}$ strain was more efficient to resist clearance by the host (Fig 7), the above result suggested greater systemic invasion by parental $S$. Typhi from the intestine. However, greater resistance of $\mathrm{A}_{200}$ strain to host clearance led to minimization of the difference between the bacterial loads of the two strains in the internal organs at $8 \mathrm{dpi}$ (Fig. 8). Together, the above results suggested that the growth of $S$. Typhi L-form was similar to the parental bacteria after oral and intraperitoneal infection, in contrast to its slower growth in thein vitro cultures. However, L-form was less efficient in systemic translocation from the gut, but resisted clearance by the host more efficiently.

\section{Discussion}

Antibiotic treatment cures bacterial infection either by killing the pathogen or halting their growth, leading to clearance of bacteria by the host immune system. However, antibiotic 
concentration in the tissues does not reach the optimum level after the first dose of the drug, rather it increases with time till it reaches the desired concentration. Thus, bacterial population within tissues is subjected to a progressively increasing gradient of antibiotic. In this study, we aimed to investigate the effects of an increasing concentration gradient of the antibiotic drug Ampicillin on Salmonella Typhi. Ampicillin inhibits transpeptidases and kills bacteria by blocking cell-wall synthesis. When ampicillin-sensitive $S$. Typhi was subjected to progressively higher doses of the drug over a period of 14 days, the bacteria changed the morphology, shut down cell wall and LPS synthesis and was able to grow in the presence of $200 \mathrm{ug} / \mathrm{ml}$ of ampicillin. This phenomenon was not observed when $S$. Typhi Ty2 strain was cultured in the presence of $200 \mathrm{ug} / \mathrm{ml}$ ampicillin from the beginning that killed all the bacterial cells. This contrasts with Salmonella Typhimurium, where L-form was induced by culturing antibiotic-sensitive bacteria with another cell wall inhibiting drug called cefazolin at a much lower dose than the MIC from the beginning [23]. Increasing concentration gradient of ampicillin led to the transition of $S$. Typhi Ty2 strain to an L-form bacteria, as characterized by drastically reduced cell wall and LPS production, an elongated shape with higher cytoplasmic volume and more than one nucleoid per cell, and significantly compromised growth in the in vitro cultures. However, characteristics of the parental strain were largely restored when ampicillin was removed from the culture media, indicating that the changes were reversible. The morphological changes observed in this study were similar to that observed for cefazolininduced L-form of S. Typhimurium [23]. Similar phenotypic changes were reported by studies with MDR E. coli A9 strain subjected to 20X MIC of ampicillin [29].

L- form of several pathogenic species were isolated from different organs of individuals suffering from symptomatic infections as well as the carriers of the disease [30]. Isolation of the L-forms from the carriers particularly fascinated the microbiologists for several decades about the actual role of this form in the pathogenesis of a disease. Streptococcus pyogenes L- 
form was found to infect an animal model and was shown to persist over a longer period of time compared with the walled form. This L-form bacterium was phagocytosed by macrophages and survived and replicated inside these cells [31]. On the contrary, L-form of another gram positive bacterium, Corynebacterium diphtheriae failed to cause productive infection and disease in the animal model. The authors concluded that defects in the cell wall formation abrogated diphtheria toxin production, leading to loss of infectivity [32]. Unlike the Gram positive bacteria, pathogenesis of Gram negative bacterial L-forms remains largely unexplored.

In this study, $S$. Typhi L- form was shown to infect murine as well as human macrophage cell lines. Gentamycin assay and confocal microscopic studies revealed active multiplication of $\mathrm{A}_{200}$ strain inside these cells. Moreover, infectivity of $\mathrm{A}_{200}$ strain was higher compared with parental $S$. Typhi. This may be explained by reduced LPS synthesis by the $\mathrm{A}_{200}$ strain, since LPS was earlier reported to inhibit phagocytosis by macrophages. However, higher infectivity of the L-form did not result in greater cell death, suggesting the potential for longer intracellular persistence.

$\mathrm{A}_{200}$ strain was equally efficient as the parental $S$. Typhi strain to establish infection in a mouse model. When administered IP, equivalent number of the bacterial strains was isolated from different organs at 2 and $4 \mathrm{dpi}$, indicating that both the strains infected and multiplied with equal efficiently in vivo. However, despite significant reduction in bacterial load at 8 dpi, suggesting bacterial clearance by the host immune system, the load for $\mathrm{A}_{200}$ strain in the host tissues remained considerably higher compared with the parental bacteria. This suggested that $\mathrm{A}_{200}$ was more efficient in evading the host immune system. This is surprising given that cell wall confers protection to bacteria against the host machinery. However, similar results were observed for Streptococcus pyogenes infection, where the L-form persisted for a longer period in the tissues and was thought to be responsible for recurrent infection by the bacteria [31]. 
Perhaps, absence of cell wall helped the L-form bacteria to avoid activation of the host immune system, thereby evading a clearance response by the host.

Surprisingly, $\mathrm{A}_{200}$ displayed different behavior inside the host upon oral delivery when compared with IP administration. Small, but equal numbers of the parental and $\mathrm{A}_{200}$ strains were isolated from different organs of mice at $2 \mathrm{dpi}$, indicating equally efficient gut colonization and early translocation to the systemic sites. However, at 4 dpi, recovery of $A_{200}$ strain was less than the parental bacteria. This was not due to slower replication of $A_{200}$ strain, as proved by the results under Fig 7. This was not due to more rapid clearance of $S$. Typhi Lform from the systemic circulation either, since both the strains were isolated at higher numbers at 8 dpi than 4 dpi. Instead, cell-wall deficient $\mathrm{A}_{200}$ strain could have activated the intestinal immune system to a greater magnitude than the parental strain, thus preventing systemic translocation from the intestine at later time points. The exact mechanisms underlying different relative recovery of the parental and L-form $S$. Typhi after oral infection requires further investigation.

$S$. Typhi was earlier shown to spontaneously convert to L-form in the gall bladder [33]. In our study, $\mathrm{A}_{200}$ strain was able to colonize the gall bladder after both oral and intraperitoneal infections. Gall bladder colonization and faecal shedding are the most important factors behind $S$. Typhi transmission from otherwise healthy carriers [34]. The prevailing concept is that $S$. Typhi shed in the stool needs to revert back to the walled phenotype to become infective for a new host. Our study showed that $S$. Typhi L-form was equally capable to cause infection, which suggests more potent human to human transmission.

\section{Conclusions}

No studies on the pathogenesis of the L-forms Salmonella spp are currently available. We, for the first time, studied pathogenesis of Salmonella Typhi L-form after oral as well as intraperitoneal infection. This study revealed that exposure of Salmonella Typhi to an increasing 
concentration gradient of ampicillin during the treatment of human infection fails to clear the entire bacterial population from the host. Rather, it may render Salmonella resistant to the antibiotic without notable genetic mutations. In this state, cell-wall deficient and elongated bacteria infects macrophages and multiply efficiently in vivo.

\section{References}

1. John Wain, Rene S Hendriksen, Matthew L Mikoleit, Karen H Keddy \& R Leon Ochiai, Typhoid fever,The Lancet, 385, Issue 9973,2015,Pages 1136-1145

2. Rowe, B., Ward, L. R., \& Threlfall, E. J. (1997). Multidrug-resistant Salmonella typhi: a worldwide epidemic. Clinical Infectious Diseases, 24(Supplement_1), S106-S109.

3. Rowe, B., Ward, L. R., \& Threlfall, E. J. (1990). Spread of multiresistant Salmonella typhi. Spread of multiresistant Salmonella typhi., 336(Oct. 27).

4. Threlfall, E. J., \& Ward, L. R. (1999). Ciprofloxacin-resistant Salmonella typhi and treatment failure. The Lancet, 353(9164), 1590-1591.

5. Qamar, F. N., Yousafzai, M. T., Khalid, M., Kazi, A. M., Lohana, H., Karim, S., ... \& Hasan, R. (2018). Outbreak investigation of ceftriaxone-resistant Salmonella enterica serotype Typhi and its risk factors among the general population in Hyderabad, Pakistan: a matched case-control study. The Lancet Infectious Diseases, 18(12), 13681376.

6. Yousafzai, M. T., Qamar, F. N., Shakoor, S., Saleem, K., Lohana, H., Karim, S., ... \& Hasan, R. (2019). Ceftriaxone-resistant Salmonella Typhi outbreak in Hyderabad City of Sindh, Pakistan: high time for the introduction of typhoid conjugate vaccine. Clinical Infectious Diseases, 68(Supplement_1), S16-S21.

7. Chatham-Stephens, K., Medalla, F., Hughes, M., Appiah, G. D., Aubert, R. D., Caidi, H., ... \& Friedman, C. R. (2019). Emergence of extensively drug-resistant Salmonella Typhi infections among travelers to or from Pakistan-United States, 2016-2018. Morbidity and Mortality Weekly Report, 68(1), 11. 
8. Krishnan, P., Stalin, M., \& Balasubramanian, S. (2009). Changing trends in antimicrobial resistance of Salmonella enterica serovar typhi and salmonella enterica serovar paratyphi A in Chennai. Indian Journal of Pathology and Microbiology, 52(4), 505.

9. Harish, B. N., \& Menezes, G. A. (2011). Preserving efficacy of chloramphenicol against typhoid fever in a tertiary care hospital, India. In Regional Health Forum (Vol.15, No. 1, p. 93).

10. Kumar, Y., Sharma, A., \& Mani, K. R. (2011). Re-emergence of susceptibility to conventionally used drugs among strains of Salmonella Typhi in central west India. The Journal of Infection in Developing Countries, 5(03), 227-230.

11. Parkhill, J., Dougan, G., James, K. D., Thomson, N. R., Pickard, D., Wain, J., ... \& Barrell, B. G. (2001). Complete genome sequence of a multiple drug resistant Salmonella enterica serovar Typhi CT18. Nature, 413(6858), 848-852.

12. Klieneberger, E. (1935). The Natural Occurrence of Pleuro-Pneumonia-like Organisms in Apparent Symbiosis with Streptobacillus moniliformis and Other Bacteria. Journal of Pathology and Bacteriology, 40, 93-105.

13. Kawai, Y., Mercier, R., Wu, L. J., Domínguez-Cuevas, P., Oshima, T., \& Errington, J. (2015). Cell growth of wall-free L-form bacteria is limited by oxidative damage. Current Biology, 25(12), 1613-1618.

14. Gilpin, R. W., Young, F. E., \& Chatterjee, A. N. (1973). Characterization of a stable Lform of Bacillus subtilis 168. Journal of Bacteriology, 113(1), 486-499.

15. Kawai, Y., Mercier, R., Mickiewicz, K., Serafini, A., de Carvalho, L. P. S., \& Errington, J. (2019). Crucial role for central carbon metabolism in the bacterial L-form switch and killing by $\beta$-lactam antibiotics. Nature microbiology, 4(10), 1716-1726.

16. Godzeski, C. W., Brier, G., Griffith, R. S., \& Black, H. R. (1965). Association of 
bacterial L-phase organisms in chronic infections. Nature, 205(4978), 1340-1340.

17. Gutman, L. T., Turck, M., Petersdorf, R. G., \& Wedgwood, R. J. (1965). Significance of bacterial variants in urine of patients with chronic bacteriuria. The Journal of clinical investigation, 44(12), 1945-1952.

18. Guze, L. B., \& Kalmanson, G. M. (1964). Persistence of bacteria in" protoplast" form after apparent cure of pyelonephritis in rats. Science, 143(3612), 1340-1341.

19. Wittler, R. G., Malizia, W. F., KRAMER, P. E., Tuckett, J. D., Pritchard, H. N., \& Baker, H. J. (1960). Isolation of a Corynebacterium and its transitional forms from a case of subacute bacterial endocarditis treated with antibiotics. Microbiology, 23(2), 315-333.

20. Almenoff, P. L., Johnson, A., Lesser, M., \& Mattman, L. H. (1996). Growth of acid fast L forms from the blood of patients with sarcoidosis. Thorax, 51(5), 530-533.

21. Nelson, E. L., \& Pickett, M. J. (1951). The recovery of L forms of Brucella and their relation to Brucella phage. The Journal of infectious diseases, 226-232.

22. Onwuamaegbu, M. E., Belcher, R. A., \& Soare, C. (2005). Cell wall-deficient bacteria as a cause of infections: a review of the clinical significance. Journal of International Medical Research, 33(1), 1-20.

23. Yang, C., Li, H., Zhang, T., Chu, Y., Zuo, J., \& Chen, D. (2020). Study on antibiotic susceptibility of Salmonella typhimurium L forms to the third and forth generation cephalosporins. Scientific reports, 10(1), 1-5.

24. Kita, E., Nishikawa, F., Kamikaidou, N., Oku, D., Yasui, K., \& Kashiba, S. (1992). Mechanism of the protective immunity against murine typhoid: persistence of Salmonella $\mathrm{L}$ forms in the liver after immunization with live-cell vaccines. FEMS microbiology immunology, 5(4), 191-199.

25. Hassan, M., Ali, A., Ahmad, A., Saleemi, M. K., Wajid, M., Sarwar, Y., \& Iqbal, M. (2021). Purification and Antigenic Detection of Lipopolysaccharides of Salmonella 
enterica Serovar Typhimurium Isolate from Faisalabad, Pakistan. Pakistan Veterinary Journal, 41(3).

26. Maleehuan, A., Ounjai, P., Harnvoravongchai, P., Janvilisri, T., \& Chankhamhaengdecha, S. (2021). Induction of Clostridioides difficile L-form.

27. Dasgupta, S., Das, S., Biswas, A., Bhadra, R. K., \& Das, S. (2019). Small alarmones (p) ppGpp regulate virulence associated traits and pathogenesis of Salmonella enterica serovar Typhi. Cellular microbiology, 21(8), e13034.

28. Stepanova, L. K., Gorelov, A. L., Sergeeva, N. S., Levina, G. A., \& IuA, B. (1983). Immunoelectrophoretic analysis of the antigenic composition of Salmonella typhi in the process of $\mathrm{L}$ transformation and reversion. Zhurnal mikrobiologii, epidemiologii, $i$ immunobiologii, (10), 29-32.

29. C Uzoechi, S., \& I Abu-Lail, N. (2020). Variations in the morphology, mechanics and adhesion of persister and resister E. coli cells in response to ampicillin: AFM study. Antibiotics, 9(5), 235.

30. Errington, J., Mickiewicz, K., Kawai, Y., \& Wu, L. J. (2016). L-form bacteria, chronic diseases and the origins of life. Philosophical Transactions of the Royal Society B: Biological Sciences, 371(1707), 20150494.

31. Michailova, L., Markova, N., Radoucheva, T., Stoitsova, S., Kussovski, V., \& Jordanova, M. (2000). Atypical behaviour and survival of Streptococcus pyogenes L forms during intraperitoneal infection in rats. FEMS Immunology \& Medical Microbiology, 28(1), 55-65.

32. Xu, Y. I., \& He, W. A. N. G. (2006). Effect of cell wall defect on pathogenicity of Corynebacterium diphtheriae. Journal of Microbiology, 01.

33. Wang, D. N., Wu, W. J., Wang, T., Pan, Y. Z., Tang, K. L., She, X. L., \& Wang, H. (2015). Salmonella L-forms: formation in human bile in vitro and isolation culture from patients' gallbladder samples by a non-high osmotic isolation technique. Clinical 
Microbiology and Infection, 21(5), 470-e9.

34. Khanam, F., Darton, T. C., Meiring, J. E., Sarker, P. K., Biswas, P. K., Bhuiyan, M. A. I., ... \& Qadri, F. (2021). Salmonella Typhi stool shedding by enteric fever patients and asymptomatic chronic carriers in an endemic urban setting. Journal of Infectious Diseases. 
A

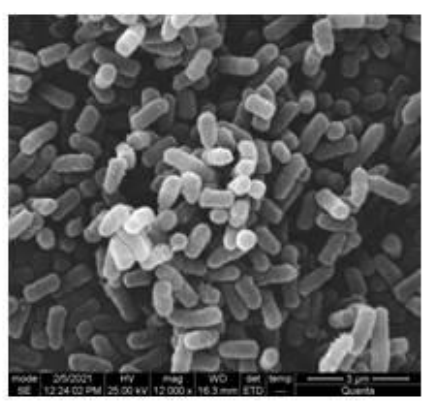

$\mathrm{D}$

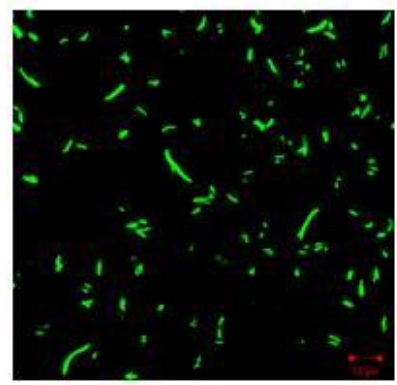

B

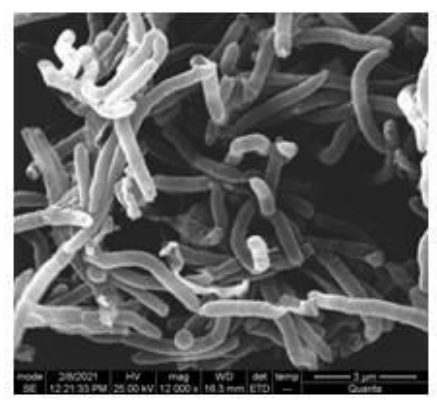

$\mathrm{E}$

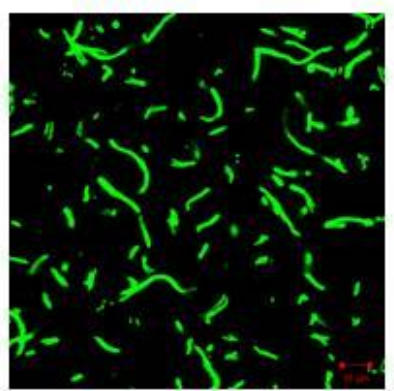

$\mathrm{C}$

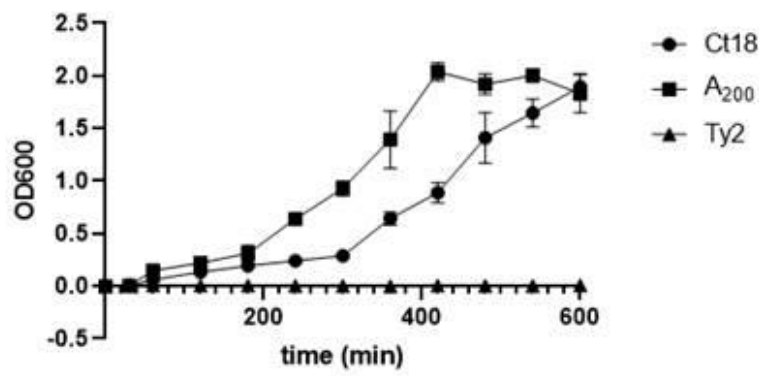

F

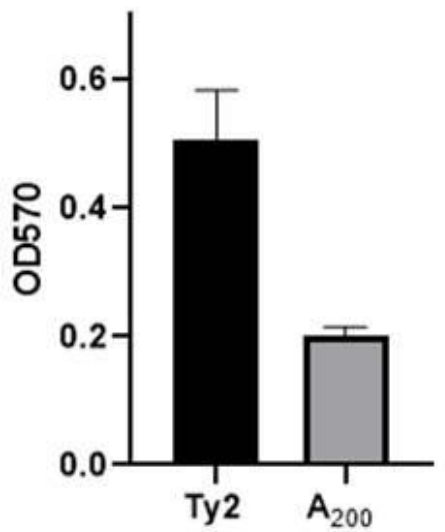

Figure 1: General characterization of A200: (A) Electron microscopy image of overnight grown WT Ty2. (B) Electron microscopy image of overnight grown $A_{200}$. (C) Growth curve comparison of Ampicillin sensitive (Ty2), Ampicillin resistant (Ct18) and $\mathrm{A}_{200} \mathrm{~L}$ form. Data represents the mean of three independent experiments with SD value indicated by the error bar. (D) Confocal microscopy of GFP containing $\mathrm{A}_{200}$ cells grown overnight in the absence of Ampicillin. (E) Confocal microscopy of GFP containing $\mathrm{A}_{200}$ cells grown overnight in the presence of Ampicillin. (F) Quantification of total peptidoglycan in Ty2 and $\mathrm{A}_{200}$ strains. Data shown as mean of three independent experiments with SD value shown by error bar 
A

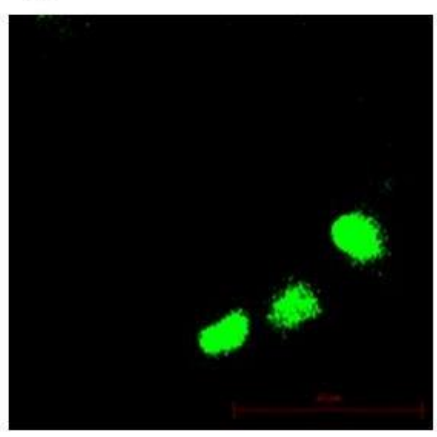

E

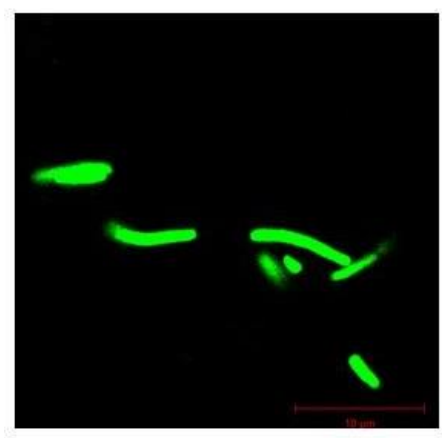

$\mathrm{F}$

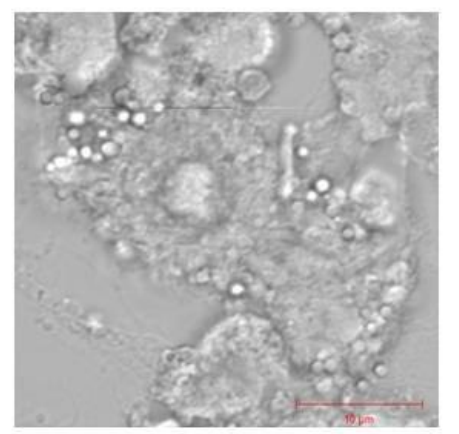

$\mathrm{C}$

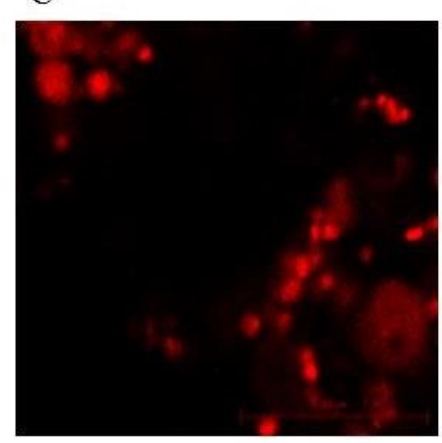

G

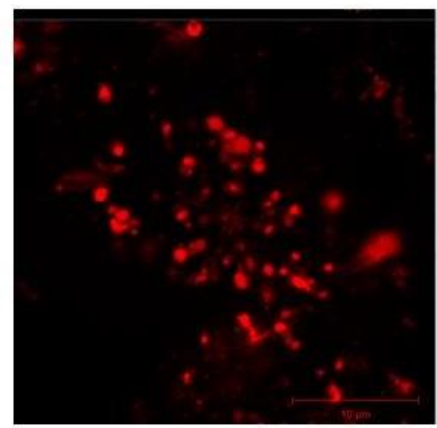

$\mathrm{D}$

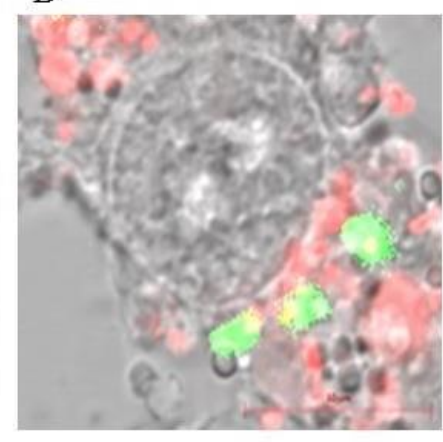

$\mathrm{H}$

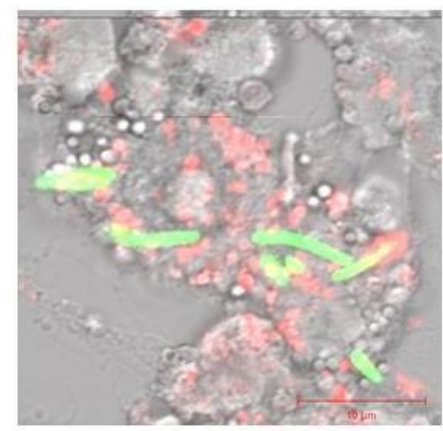

Figure 2. Infection of murine RAW cell line with GFP- Ty2 and GFP- A200 L-form at 0 hr: Representative confocal microscopy images showing intracellular Ty2 (upper panel) and $\mathrm{A}_{200}$ (lower panel) bacteria at 0hr post infection. (A and E) GFP containing bacteria. (B and F) DIC image of RAW cells. (C and G) Acidic vacuoles stained with Dextran Alexa Fluor. (D and H) Merge of all the above filter 
A

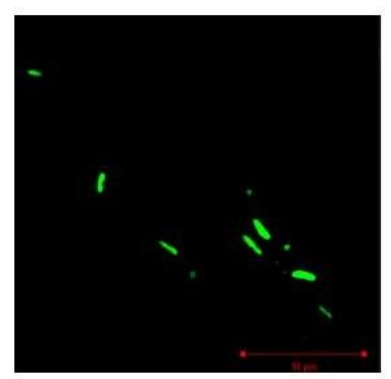

$\mathrm{E}$

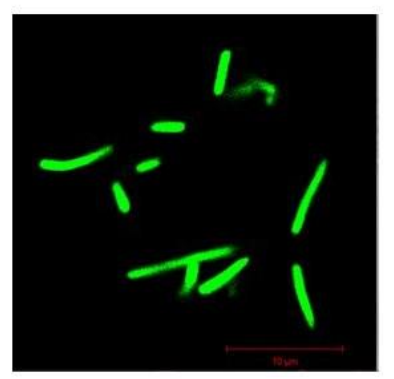

B

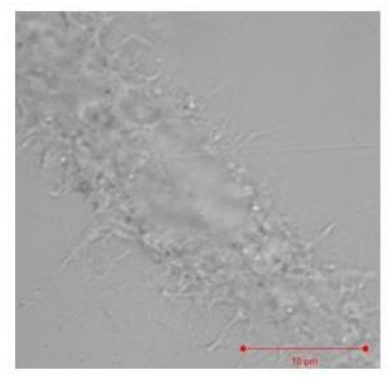

$\mathrm{F}$

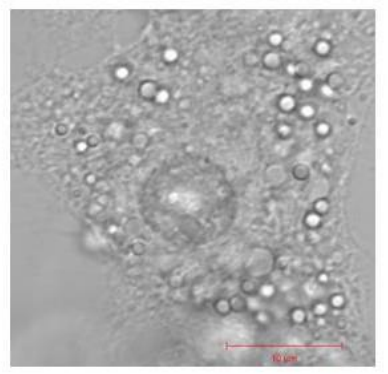

$\mathrm{C}$

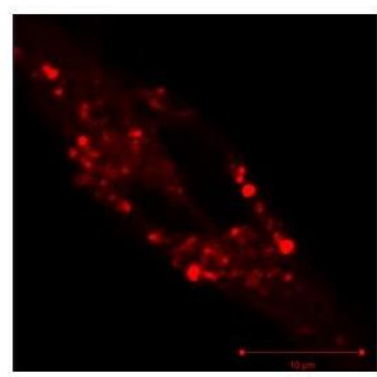

G

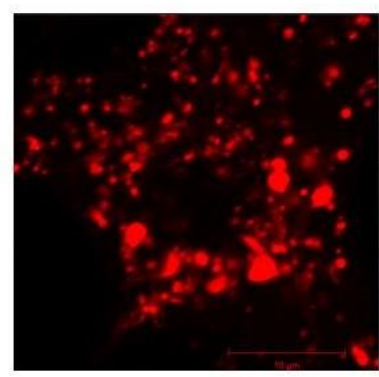

$\mathrm{D}$

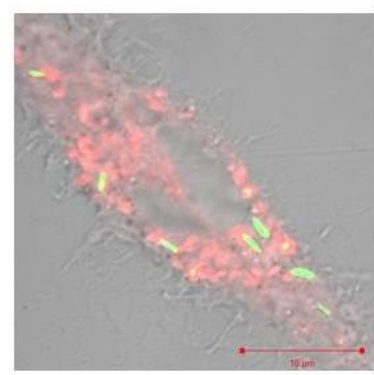

$\mathrm{H}$

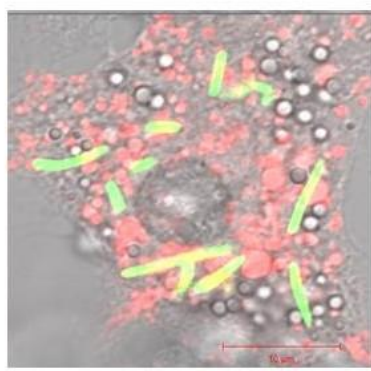

Figure 3: Infection of murine RAW cell line with GFP- Ty2 and GFP- A200 L-form at 24hr:

Representative confocal microscopy images showing intracellular Ty2 (upper panel) and $\mathrm{A}_{200}$ (lower panel) bacteria at $24 \mathrm{hr}$ post infection. (A and E) GFP containing bacteria. (B and F) DIC image of RAW cells. (C and G) Acidic vacuoles stained with Dextran Alexa Fluor. (Dand H) Merge of all the above filters. 


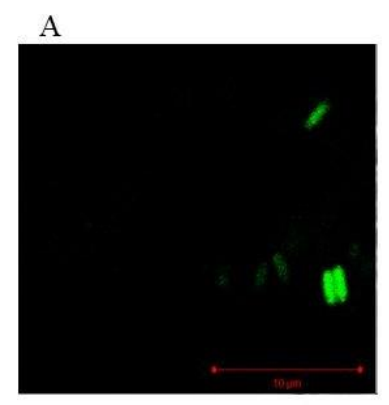

E

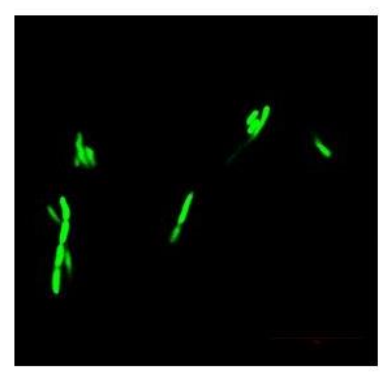

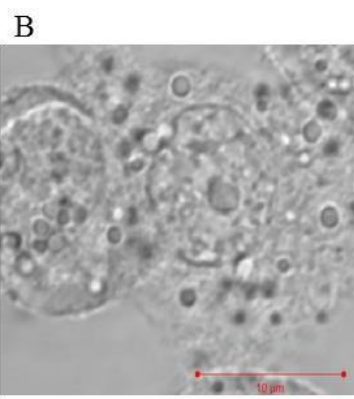

$\mathrm{F}$

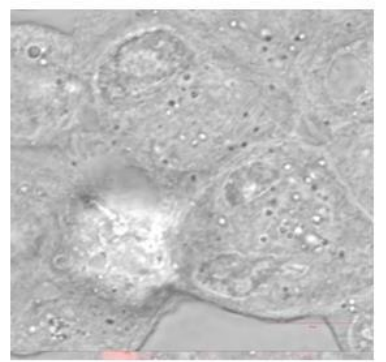

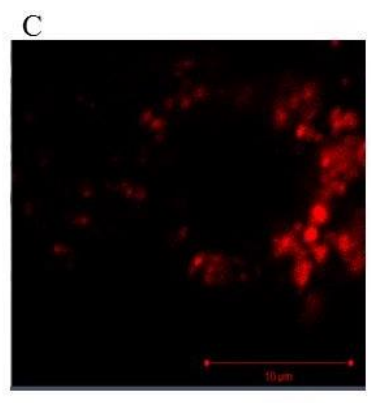

G

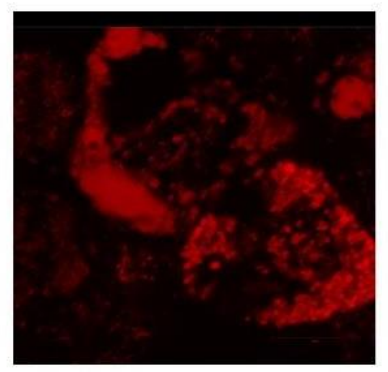

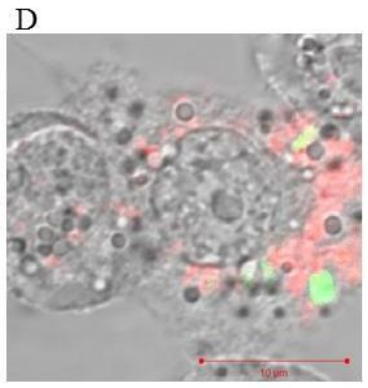

$\mathrm{H}$

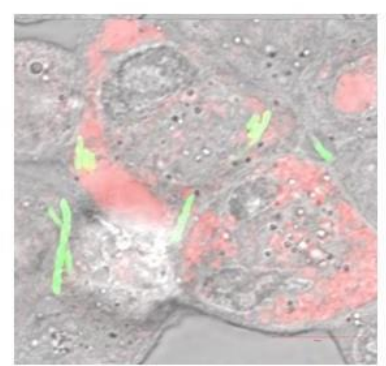

Figure 4: Infection of THP-1 cell line with GFP- Ty2 and GFP- A200 L-form at 0 hr:

Representative confocal microscopy images showing intracellular Ty2 (upper panel) and A200 (lower panel) bacteria at $0 \mathrm{hr}$ post infection. (A and E) GFP containing bacteria. (B and F) DICimage of THP1 cells. (C and G) Acidic vacuoles stained with Dextran Alexa Fluor. (D and H)Merge of all the above filters. 
A

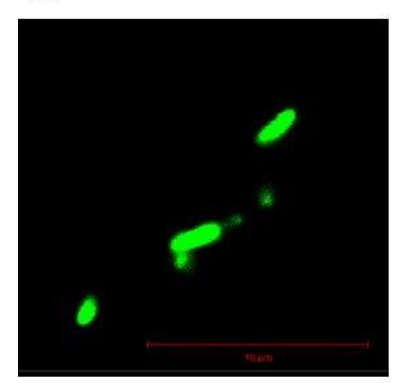

E

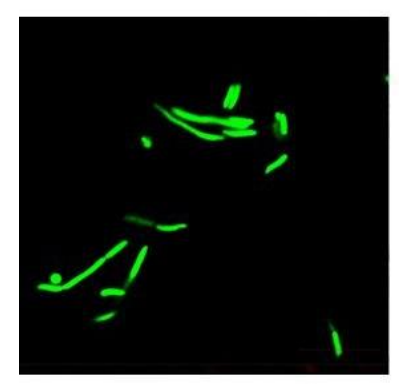

B

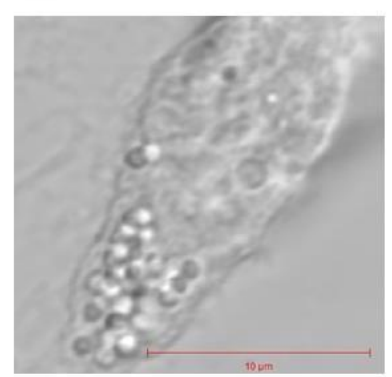

$\mathrm{F}$

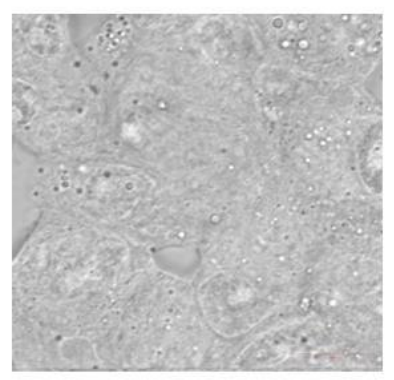

$\mathrm{C}$

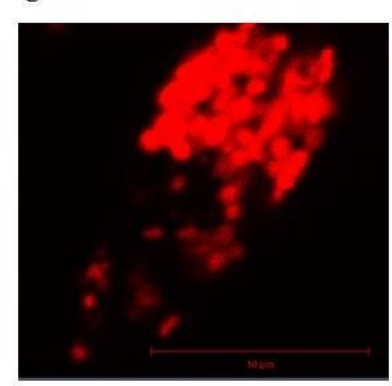

G

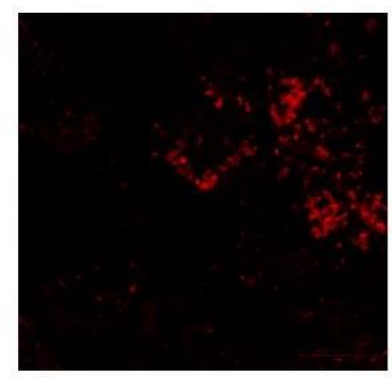

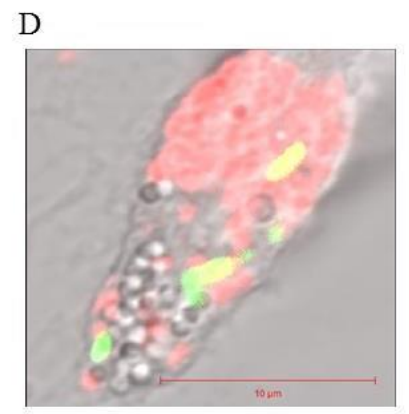

$\mathrm{H}$

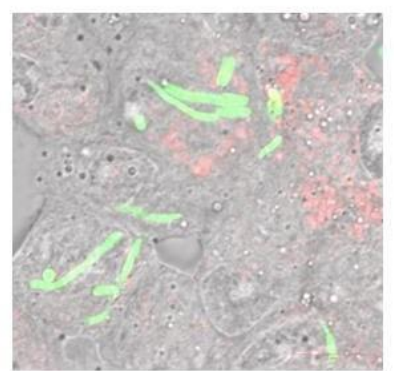

Figure 5: Infection of THP-1 cell line with GFP- Ty2 and GFP- A200 L-form at 24 hr: Representative confocal microscopy images showing intracellular Ty2 (upper panel) and A200 (lower panel) bacteria at $24 \mathrm{hr}$ post infection. (A and E) GFP containing bacteria. (B and F) DIC image of THP-1 cells. (C and G) Acidic vacuoles stained with Dextran Alexa Fluor. (D and H) Merge of all the above filters. 
A

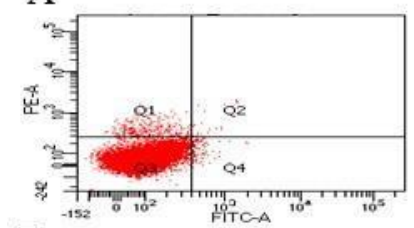

$\mathrm{D}$

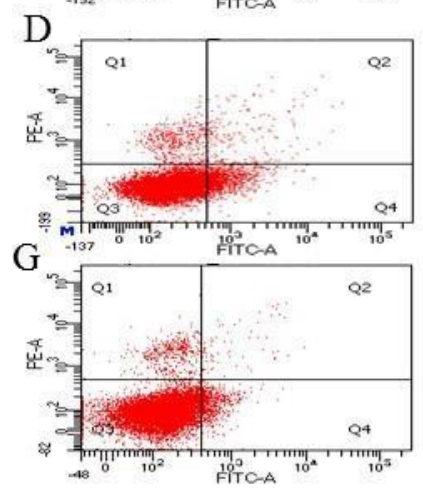

B

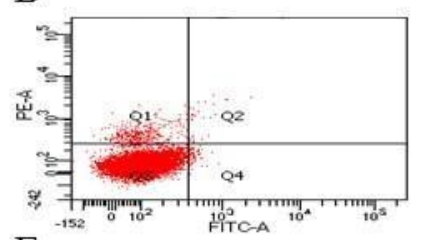

$\mathrm{E}$
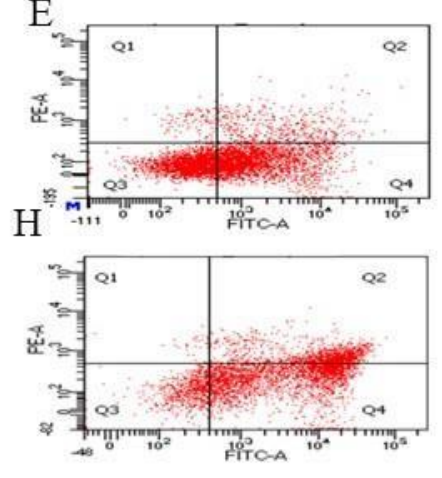

$\mathrm{J}$

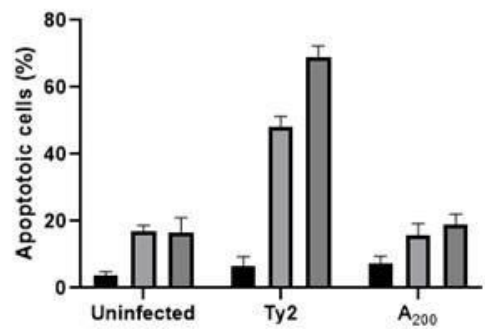

$\mathrm{C}$
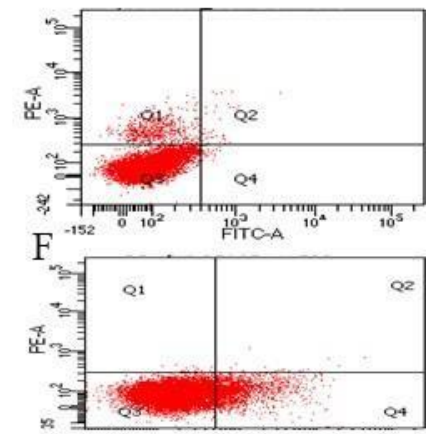

I

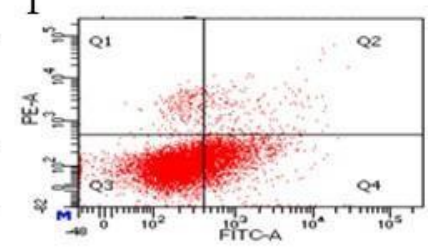

- $0 \mathrm{hr}$ post infection

ㅁ $24 \mathrm{hrs}$ post infection

口 48 hrs post infection

Figure 6: Apoptosis induced by Ty2 and A200 in THP-1 cell line: Representative flow diagram of Ty2 and $\mathrm{A}_{200}$ infected THP-1 cells. (A) uninfected THP-1 cells, 0 hr. (B) Ty2 infectedTHP-1 cells , 0 hr. (C) A 200 infected THP-1 cells , 0 hr. (D) uninfected THP1 cells , 24 hr, (E)Ty2 infected THP-1 cells , 24 hr. (F) $\mathrm{A}_{200}$ infected THP-1 cells , 24 hr. (G) Uninfected THP1cells, 48 hr (H) Ty2 infected THP1 cells , 48 hr (I) A200 infected THP1 cells, 48hr. (J) Quantification of apoptotic cell by flow cytometry after annexin V/ PI staining of treated and untreated Thp- 1 cell lines after $0 \mathrm{hr}, 24 \mathrm{hrs}$ and $48 \mathrm{hrs}$ post infection. Data represents the mean of three independent experiments with SD value indicated by the error bar. 
A

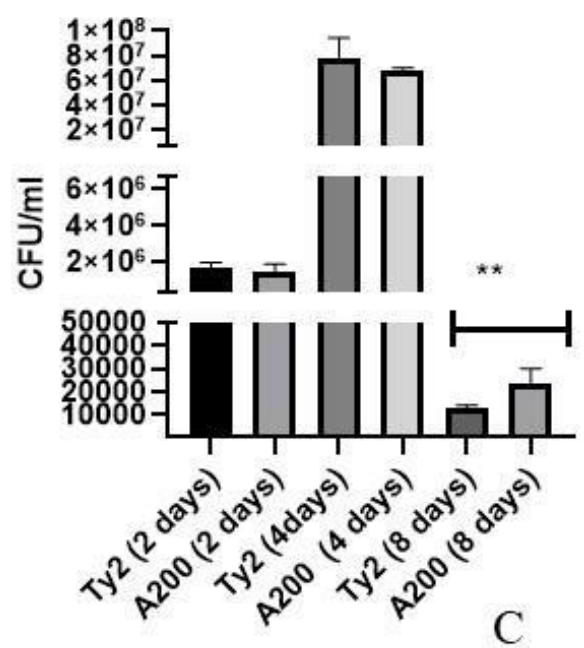

B

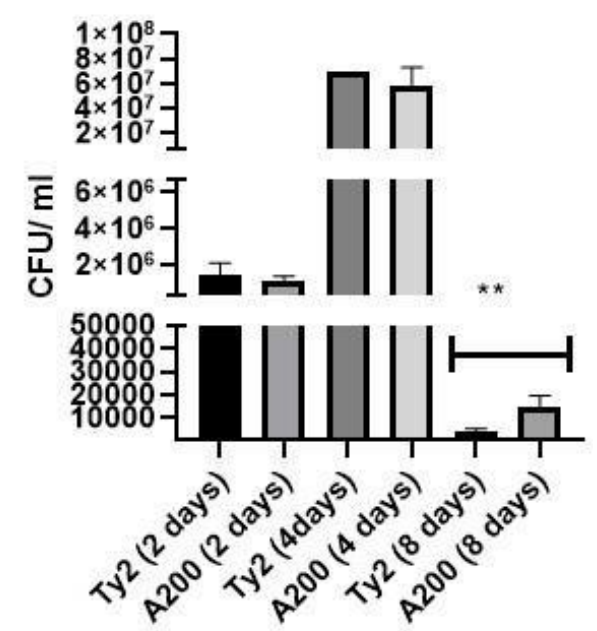

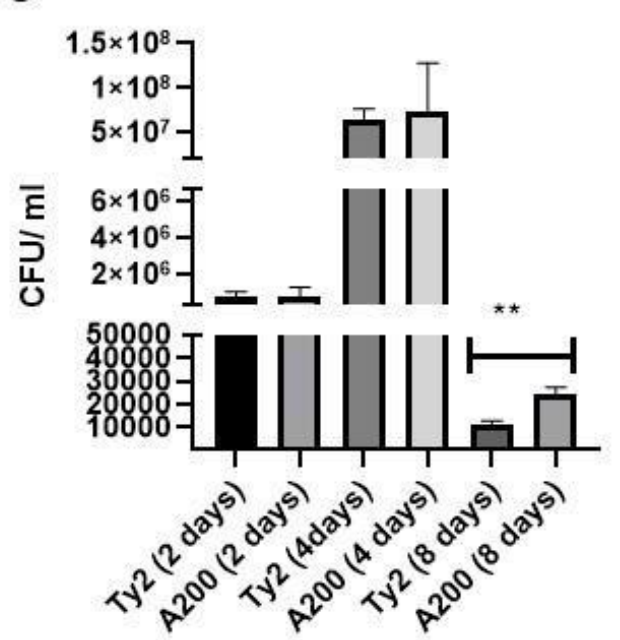

Figure 7: Infection of mice model by A200 L form (IP): Mice ( $\mathrm{n}=5)$ were infected intraperitoneally with $\mathrm{A}_{200} \mathrm{~L}$ - form. Bacteria was isolated from different organs, (A) liver, (B) spleen, (C) Gall bladder after 2, 4, and 8 dpi and platted on LA plates. CFU was calculated and plotted. Data represents the mean of three independent experiments with SD value indicatedby the error bar. 
A

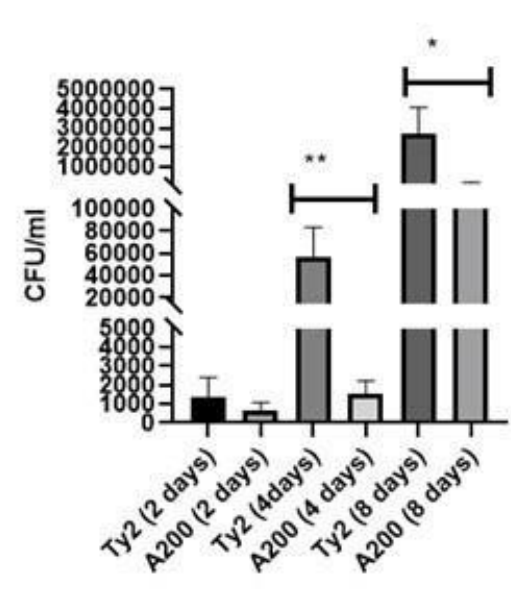

B

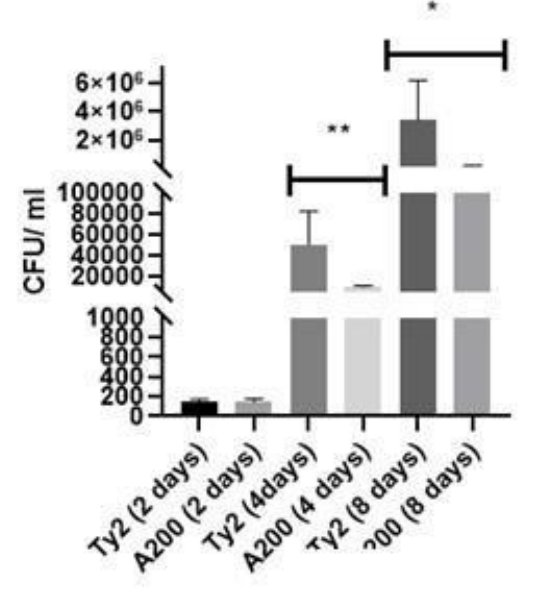

C

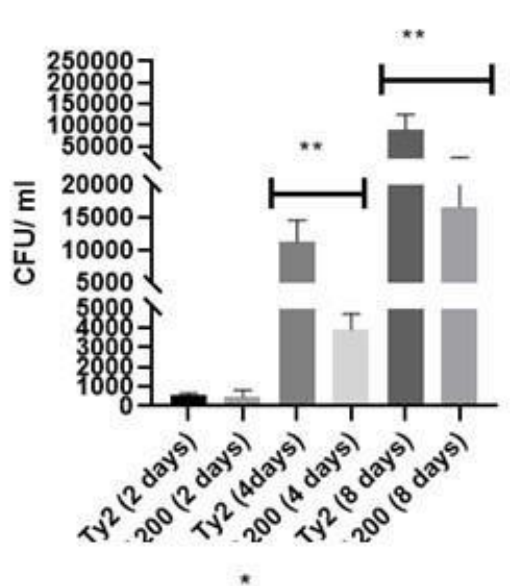

D

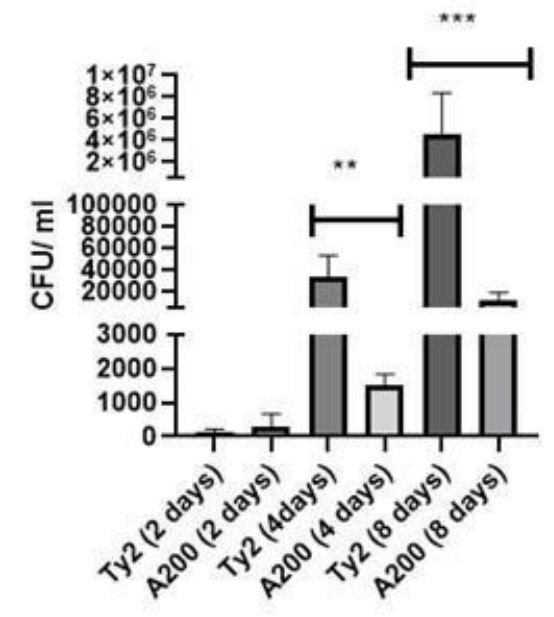

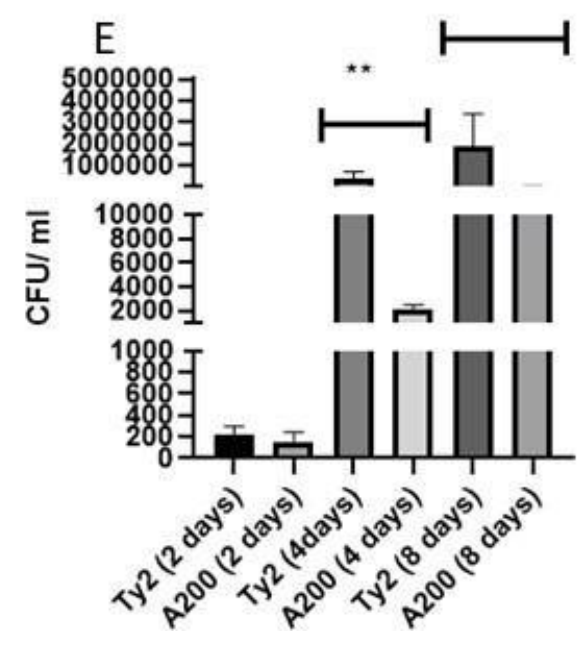

Figure 8: Infection of mice model by $\mathbf{A}_{200} \mathbf{L}$ form (oral): Mice $(\mathrm{n}=5)$ were infected orally with $\mathrm{A}_{200}$

L- form. Bacteria was isolated from different organs, (A) liver, (B) Spleen , (C) Gall bladder, (D) MLN

, (E) Payers' patch after 2, 4, and 8 dpi and platted on LA plates. CFU was calculated and plotted. Data represents the mean of three independent experiments with SD value indicated by the error bar. 


\section{Figure legends.}

Figure 1: General characterization of A200: (A) Electron microscopy image of overnight grown WT Ty2.

(B) Electron microscopy image of overnight grown $A_{200}$. (C) Growth curve comparison of Ampicillin sensitive (Ty2), Ampicillin resistant (Ct18) and $\mathrm{A}_{200} \mathrm{~L}$ form. Data represents the mean of three independent experiments with SD value indicated by the error bar. (D) Quantification of total peptidoglycan in Ty2 and A200 strains. Data shown as mean of threeindependent experiments with SD value shown by error bar (E) Confocal microscopy of GFP containing $A_{200}$ cells grown overnight in the absence of Ampicillin. (F) Confocal microscopy of GFP containing A200 cells grown overnight in the presence of Ampicillin.

Figure 2. Infection of murine RAW cell line with GFP- Ty2 and GFP- A200 L-form at 0 hr: Representative confocal microscopy images showing intracellular Ty2 (upper panel) and $\mathrm{A}_{200}$ (lower panel) bacteria at $0 \mathrm{hr}$ post infection. (A and E) GFP containing bacteria. (B and F)DIC image of RAW cells. (C and G) Acidic vacuoles stained with Dextran Alexa Fluor. (D and H) Merge of all the above filters.

Figure 3: Infection of murine RAW cell line with GFP- Ty2 and GFP- A200 L-form at 24hr: Representative confocal microscopy images showing intracellular Ty2 (upper panel) and A200 (lower panel) bacteria at $24 \mathrm{hr}$ post infection. (A and E) GFP containing bacteria. (B and F) DIC image of RAW cells. (C and G) Acidic vacuoles stained with Dextran Alexa Fluor. (Dand H) Merge of all the above filters.

Figure 4: Infection of THP-1 cell line with GFP- Ty2 and GFP- A200 L-form at 0 hr: Representative confocal microscopy images showing intracellular Ty2 (upper panel) and $\mathrm{A}_{200}$ (lower panel) bacteria at $0 \mathrm{hr}$ post infection. (A and E) GFP containing bacteria. (B and F) DICimage of THP-1 cells. (C and G) Acidic vacuoles stained with Dextran Alexa Fluor. (D and H)Merge of all the above filters.

Figure 5: Infection of THP-1 cell line with GFP- Ty2 and GFP- A200 L-form at 24 hr: 
Representative confocal microscopy images showing intracellular Ty2 (upper panel) and A200 (lower panel) bacteria at $24 \mathrm{hr}$ post infection. (A and E) GFP containing bacteria. (B and F) DIC image of THP-1 cells. (C and G) Acidic vacuoles stained with Dextran Alexa Fluor. (D and H) Merge of all the above filters.

Figure 6: Apoptosis induced by Ty2 and A200 in THP-1 cell line: Representative flow diagram of Ty2 and A 200 infected THP-1 cells. (A) uninfected THP-1 cells, 0 hr. (B) Ty2 infectedTHP-1 cells , 0 hr. (C) A200 infected THP-1 cells , 0 hr. (D) uninfected THP1 cells , 24 hr, (E)Ty2 infected THP-1 cells , 24 hr. (F) A200 infected THP-1 cells , 24 hr. (G) Uninfected THP1cells, 48 hr (H) Ty2 infected THP1 cells , 48 hr (I) A200 infected THP1 cells, 48hr. (J) Quantification of apoptotic cell by flow cytometry after annexin V/ PI staining of treated and untreated Thp- 1 cell lines after $0 \mathrm{hr}, 24 \mathrm{hrs}$ and $48 \mathrm{hrs}$ post infection. Data represents the meanof three independent experiments with SD value indicated by the error bar.

Figure 7: Infection of mice model by A200 L form (IP): Mice ( $\mathrm{n}=5$ ) were infected intraperitoneally with $\mathrm{A}_{200} \mathrm{~L}$ - form. Bacteria was isolated from different organs, (A) liver, (Bspleen, (C) Gall bladder after 2, 4, and 8 dpi and platted on LA plates. CFU was calculated and plotted. Data represents the mean of three independent experiments with SD value indicatedby the error bar.

Figure 8: Infection of mice model by $\mathbf{A}_{200} \mathbf{L}$ form (oral): Mice ( $\left.=5\right)$ were infected orally with A 200 L- form. Bacteria was isolated from different organs, (A) liver , (B) Spleen , (C) Gall bladder , (D) MLN , (E) Payers' patch after 2, 4, and 8 dpi and platted on LA plates. CFU was calculated and plotted. Data represents the mean of three independent experiments with SD value indicated by the error bar. 


\section{Data availability}

All data supporting the final conclusions are available in figures

\section{Conflict of interest}

The authors declare that there is no conflict of interest regarding the publication of this article

\section{Funding statement}

This project has been funded by the institute (intramural project). 